\title{
Design of Fingerprint Identification System based on FPGA
}

\author{
Kuo Fu and Yufeng Yang ${ }^{\mathrm{b}}$
}

Department of Mechanical and Electrical Engineering, Guangdong University of Science \&
Technology, Dongguan, China

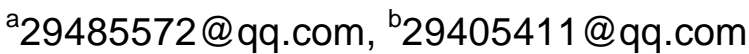

* The Corresponding Author

Keywords: Fingerprint Identification System; FPGA; Fingerprint Image; Data Communication Module

\begin{abstract}
This system is a fingerprint identification system developed in the fingerprint identification module. The system uses fingerprint module to search fingers. Once fingers are searched, fingerprint images are collected immediately, and the collected images are converted to data form to send to the FPGA. It uses the fingerprint and invariance of the anisotropy to provide encryption method for users. The fingers flat on the fingerprint collection window can complete the acquisition tasks, and display the process and results of fingerprint fingerprint image acquisition module through the LED . In addition, the required functions can be selected by the independent key.
\end{abstract}

\section{Introduction}

With the progress of optical technology and optical instrument processing technology, high precision and distortion free optical acquisition instrument is equipped with all kinds of fingerprint authentication system and equipment reached a high level, which ensures that high-quality fingerprint images can be generated. The improvement of computer computing speed and the progress of computer miniaturization make it possible to use a microcomputer or even a single chip computer to perform a fingerprint contrast operation. Modern electronic integrated manufacturing technology enables us to produce fairly small fingerprint image reading devices and fingerprint identification modules. Its cost is falling fast, which greatly accelerates the speed of the spread of fingerprint identification technology.

\section{Preprocessing of Fingerprint Image}

Normalization is a kind of each pixel of the original fingerprint image on the human operation. In order to change its gray value and eliminate the fingerprint input sensor surface residual noise and finger pressure difference of the fingerprint image, the image is divided into blocks for each block the gray mean $M_{i}$ and variance $V_{i}$. The calculation of the mean and variance of the subblock grayscale is based on the following formula,

$$
\begin{aligned}
& M_{i}=\frac{1}{w \times w} \sum_{x-1}^{w} \sum_{y-1}^{w} I(x, y) \\
& V_{i}=\frac{1}{w \times w} \sum_{x-1}^{w} \sum_{y-1}^{w}\left[I(x, y)-M_{i}\right]^{2}
\end{aligned}
$$

$\mathrm{I}(\mathrm{x}, \mathrm{y})$ is the gray value of pixels $(\mathrm{x}, \mathrm{y})$ in the sub block. The purpose of normalization is to adjust the mean and variance to a desired range. If $G(i, j)$ is used to represent the normalized value of the point ( $x$, y), then the normalized value of the I block is normalized.

$$
G(i, j)=M_{0} \pm \sqrt{\frac{V_{0}\left(I(x, y)-M_{i}\right)}{V_{i}}}
$$

Among them, $M_{0}$ is the mean and variance of expectations, $M$ is the threshold value obtained after experiments, $I(i, j)-M i$ is gray pixel amplitude. The original point of the normalized variance was 
larger whereas the opposite, and gray value is less than the mean value of the point, which will not change the texture structure of fingerprint.

The fingerprint image is divided into foreground, background area, fuzzy areas and white background, the white background region refers to the boundary region does not contain the fingerprint in the fingerprint image, this area is also should be included in the cut area, so in order to correctly divide the 4 regions more accurately cut off white background and the background area, regional recovery and retain the foreground and fuzzy region, the segmentation process is divided into 3 levels, the basic unit for segmentation of overlapping block sizes. The first stage is divided into second levels of white background segmentation, segmentation is a fuzzy area, level third is divided into background segmentation, white background gray value is very low, and because of the change of no ridge peaks and valleys, the variance is very small, so the white background is judged by block gray mean and block gray variance

$$
\begin{aligned}
\bar{x} & =\frac{\sum_{i=0}^{15} \sum_{j=0}^{15} I(i+m \times 8, j+n \times 8)}{8 \times 8} \\
\sigma & =\frac{\sum_{i=0}^{15} \sum_{j=0}^{15}[I(i+m \times 8, j+n \times 8)-\bar{x}]^{2}}{8 \times 8}
\end{aligned}
$$

\section{The Hardware Design}

The system uses the Xilinx company Spartan3E series FPGA as the core of the control and operation of the chip, and the data acquisition module is completed by the Fujitsu Corporation MBF200 capacitive fingerprint sensor. MBF200 fingerprint sensor can complete the collection of fingerprint image and its own A/D converter converts the fingerprint image into digital signals by using the SPI interface transmission to FPGA. The data collected by FPGA process can not store a complete fingerprint image because FPGA's internal storage space is too small, so the fingerprint data is temporarily stored in SDRAM. When processing image data, FPGA reads the fingerprint data of SDRAM and performs operations such as filtering, grayscale normalization, two valued, thinning and eigenvalue extraction in its logic unit, so as to get important fingerprint image information.

Fingerprint Acquisition Module. This design uses Fujitsu's MBF200 fingerprint sensor. The MBF200 hardware block diagram is shown in Figure 1. The SPI mode is adopted, so MBF200 and FPGA connect to four ports, MISO, MOSI, /S/C/S and SCLK.

Fingerprint Data Processing and Storage Module. Because fingerprint data processing involves many algorithms and large amount of computation, this system embeds a soft core on the development board for data processing. First, a large number of fingerprint data transmitted from SPI port are sent to SDRAM on the development board for storage. When creating fingerprint database, the system takes data out of SDRAM and puts it into Microblaze to process. Then the processed fingerprint data is stored in FLASH to form fingerprint database. In the comparison mode, data was disposed by Microblaze after being processed, and then stored in SDRAM. Then the fingerprint image feature data were extracted from FLASH and SDRAM respectively, and the comparison results were obtained. The hardware connection of FLASH input storage module is as shown in Figure 2. 


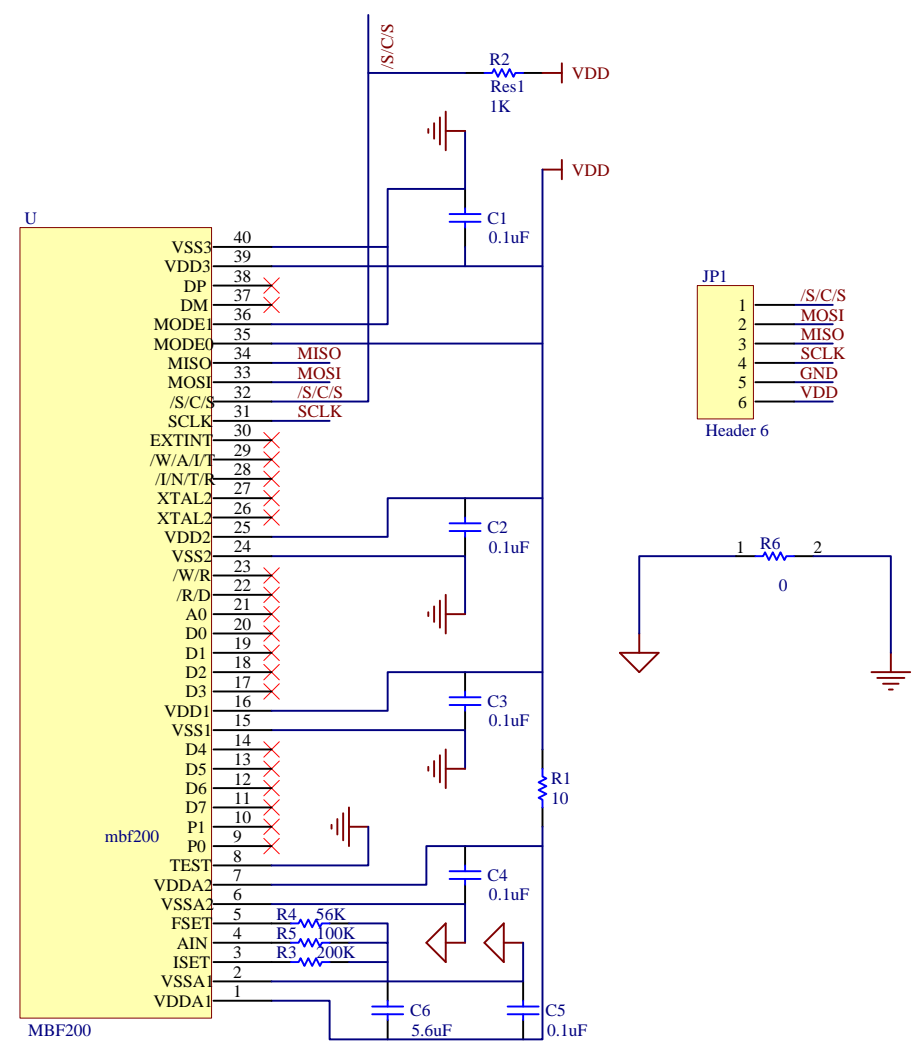

Figure 1. The MBF200 hardware connection circuit

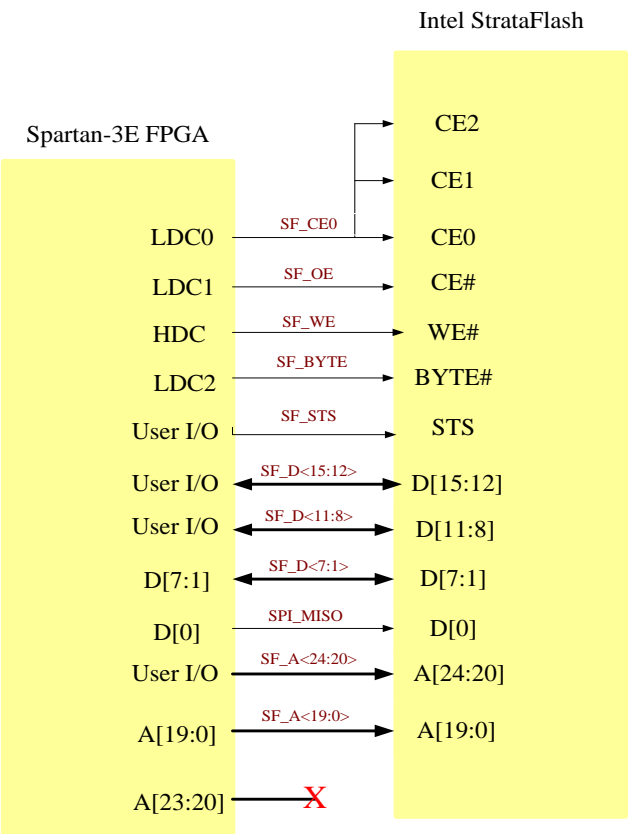

Figure 2. The hardware connection of FLASH input storage module

Fingerprint Data Communication Module. In single chip computer programming, serial port occupies a very important position. The debugging of the traditional serial port program is often used for a special MCU hardware simulator. After the program is written, the breakpoints are set by the emulator, and the flow of the variable and the program is observed. The program is debugged and the error is corrected. The serial port circuit is as shown in Figure 3. 


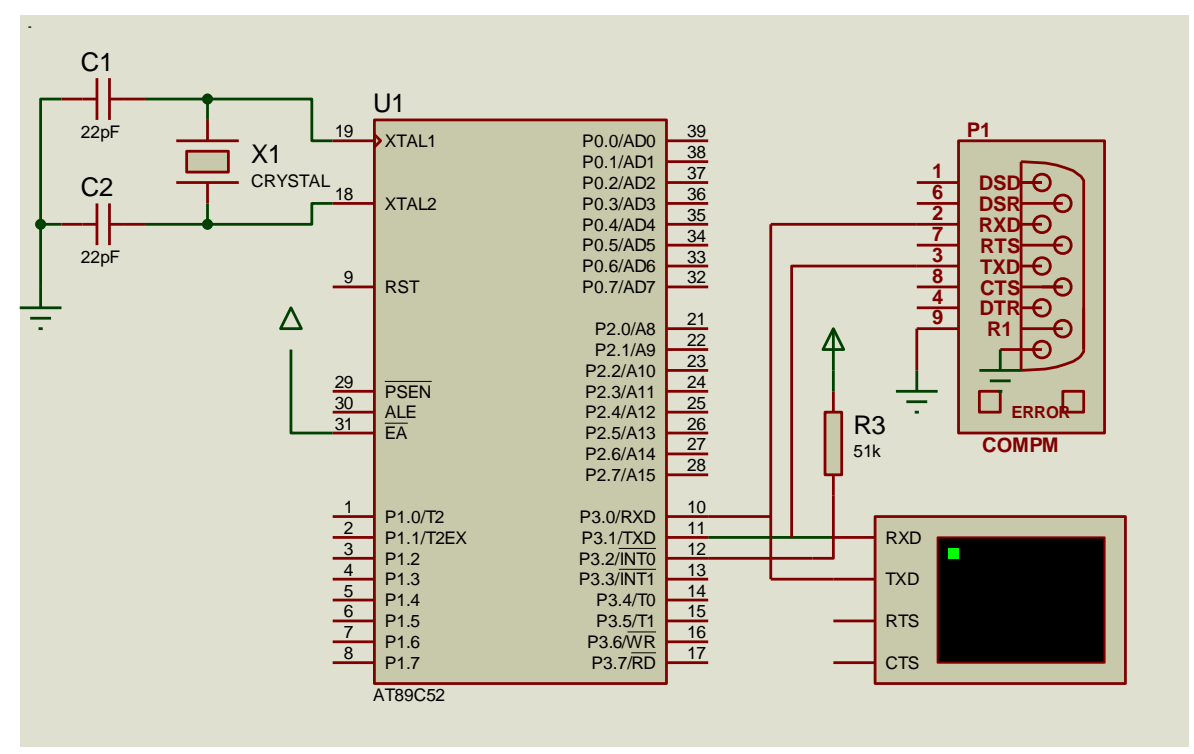

Figure 3. The serial port circuit

\section{Summary}

With the development of science and technology in making social progress, many system security problem becomes the primary consideration. This paper aims at the limitation of traditional system security and applies fingerprint identification module to design the hardware of the fingerprint identification based on MCU control. The system realizes the serial communication between the microcontroller and the fingerprint module, sends corresponding commands to fingerprint module through stand-alone keyboard, and finishes some functions, such as users addition, users deleting, user authentication, user management, permissions.

\section{References}

[1] Dutt-Mazumder M A, Button C, and Robins A. Neural Network Modelling and Dynamical System Theory, Sports Medicine, 41(2011), 1003-1011.

[2] Jahan M S, Asaduzzaman M, and Sarkar A K. Performance of Broiler Fed on Mash, Pellet and Crumble. International Journal of Poultry Science, 5(2016), 76-88.

[3] Vereecken H, and Herbst M. Statistical Regression, Developments in Soil Science, 30 (2014), 3-19.

[4] Yang Zhao,Yanguang Cai, and Defu Cheng. A Novel Local Exploiation Scheme for Conditionally Breeding Real-coded Genetic Algorithm, Multimedia Tools and Applications, 76 (2017), 17955-17969.

[5] W G Chen, S D Peng, Y Y Wang. Improved Support Vector Machine for Regression and its Application to Sensor Array Pattern Recognition, High Voltage Apparatus, 47 (2011), 82-86.

[6] Ke Ye . Prospect and Present Situation of Pellet Production in China, Iron Making, 9(2012), 65-70.

[7] Jin Li, and Sheng Jiang. Short-Term Climate Prediction Model of Neural Network Based on Genetic Algorithms, Plateau Meteorology, 24 (2015), 981-987.

[8] Yang Zhao, Zhenghong Guo, and Jianming Yan. Vibration Signal Analysis and Fault Diagnosis of Bogies of the High-speed Train based on Deep Neural Networks, Journal of Vibroengineering, 19 (2017), 2456-2474.

[9] Philiastides M G, Biele G, and Vavatzanidis N. Temporal Dynamics of Prediction Error Processing During Reward-based Decision Making, Neuroimage, 53 (2010), 221-229.

[10] S Ding, C Su, and J Yu. An Optimizing BP Neural Network Algorithm based on Genetic Algorithm, Artificial Intelligence Review, 36 (2011), 153-162. 